COMMENT. In this uncontrolled study of melatonin $(1.5 \mathrm{mg})$ in children with refractory epilepsy, sleep continuity and some sleep-related disorders were significantly improved. Seizure severity, but not frequency, also showed significant improvement.

\title{
INTERICTAL EEG SPIKES IDENTIFY SEIZURE FOCUS
}

A computerized spike detector was used to measure and localize interictal epileptiform discharges (IEDs) over prolonged, representative segments of recordings in 19 children with intractable, mostly extratemporal lobe epilepsy. Approximately 8 hours of IEEG, randomly selected 30-min segments of continuous interictal IEEG per patient, were analyzed over all intracranial electrode contacts. Spike frequency was averaged over the 16-time segments, and electrodes with the highest mean spike frequency were within the seizure-onset region in 11 of 19 patients. Large statistical samples of interictal activity were required for improved localization. Low-voltage fast EEG at seizure onset was the only factor predicting IED localization to the seizure-onset region. Automated IED detection over multiple samples of IEEG may be of value in planning epilepsy surgery for intractable epilepsy in children. (Marsh ED, Peltzer B, Brown MW, et al. Interictal EEG spikes identify the region of electrographic seizure onset in some, but not all, pediatric epilepsy patients. Epilepsia 2010;51(4):592-601). (Respond: Eric D Marsh MD PhD, Division of Child Neurology, Children's Hospital of Philadelphia, 3400 Civic Center Blvd, Philadelphia, PA 19104. E-mail: marshe@email.chop.edu).

COMMENT. Ictal recording is the mainstay of localizing epileptic foci for surgical resection. The inconsistent correlation with interictal spikes in previous reports may be related to the relatively short periods of observation. Further research is recommended to determine which patients may benefit from this technique.

\section{VASCULAR DISORDERS}

\section{MINOR ATHLETIC TRAUMA AND ISCHEMIC STROKE}

Three cases of arterial ischemic stroke (AIS) following sports-related neck trauma are reported from Johns Hopkins University School of Medicine, Baltimore, MD. Case I. A 10-year-old boy developed a left hemiparesis within a few minutes of colliding with another soccer player and sustaining a hit on his right head and neck. MRI revealed a right basal ganglia stroke. MRA showed no evidence of arterial dissection, and echocardiogram was normal. Treatment with heparin (UFH) followed by aspirin resulted in complete recovery. He was heterozygous for factor V mutation and MTHFR C677T polymorphism. Case 2. A 12-year-old boy sustained right neck and shoulder trauma during a lacrosse game. Later that night, he developed tingling of his right face and headache, and the next morning he awoke with vomiting, right-sided weakness, followed by nystagmus, truncal ataxia, and dysmetria of the right upper and lower limbs. MRI confirmed stroke in the right posterior pontomedullary junction. Traumatic vertebral artery dissection was suspected, and heparin was administered. Digital subtraction angiography (DSA) performed $12 \mathrm{~h}$ after admission showed no dissection, and heparin 